\title{
An analysis of the solar-like oscillations in the red giant star KIC 5701829 observed with Kepler
}

\author{
Lester Fox-Machado ${ }^{1}$ and Dan Deras ${ }^{2}$ \\ ${ }^{1}$ Instituto de Astronomía, Universidad Nacional Autónoma de México, \\ Ap. P. 877, Ensenada, BC 22860, México \\ email: lfox@astro.unam.mx \\ ${ }^{2}$ Facultad de Ciencias, Universidad Nacional Autónoma de México \\ email: danj.deras@gmail.com
}

\begin{abstract}
The preliminary results of an analysis of the red giant star KIC 5701829 observed for 29 days in short-cadence mode with the Kepler satellite are reported. The oscillation spectrum of this star is characterized by the presence of a well-defined solar-like oscillation pattern due to acoustic modes. The characterization of the power spectrum has been performed following three basics steps commonly used in the analysis of solar-like oscillations: fitting and correcting for the background, estimating the frequency of maximum power $\left(\nu_{\max }\right)$ and the large separation $(\Delta \nu)$, and extracting individual frequencies. We have found that the frequency of maximum oscillation power, $\nu_{\max }$, and the mean large frequency separation, $\Delta \nu$, are around, 143 and 12 $\mu \mathrm{Hz}$, respectively. The global asteroseismic parameters along with atmospheric parameters from the literature allow us to infer about evolutionary status of the star.
\end{abstract}

Keywords. stars: oscillations, stars: individual (KIC 5701829)

\section{Introduction}

The Kepler satellite (Borucki et al. 2010), successfully launched in 2009 March, is providing light curves of impressive quality which are specially well suited for probing the interior of the stars by using the techniques of asteroseismology (Aerts et al. 2010). During the first three years of the mission Kepler was observing continuously a fixed fieldof-view (FOV) of $\sim 105$ square degrees in the constellations Cygnus and Lyra. This FOV contains a huge number of intrinsic pulsator of different types for which the analysis of the high precision Kepler light curves yields an important improvement in our understanding of the structure and evolution of the stars (e.g. Uytterhoeven et al. 2011). In particular, asteroseismology of red giant stars has been developed very fast in the Kepler era. As the pulsations observed in red giant stars are stochastically excited by the turbulent covection in the upper layers, their oscillation spectrum is characterized by the presence of a well-defined solar-like oscillation pattern due to acoustic modes. The analysis of the power spectrum of thousand red giant stars observed by Kepler has provided precise characterization of their overall characteristics and, in some cases, detailed information on the properties their deep interiors (e.g Hekker et al. 2011, Bedding et al. 2010, Di Mauro et al. 2011, Beck et al. 2014 ). In this paper we present a preliminary analysis of the KIC 5701829 Kepler light curve. 

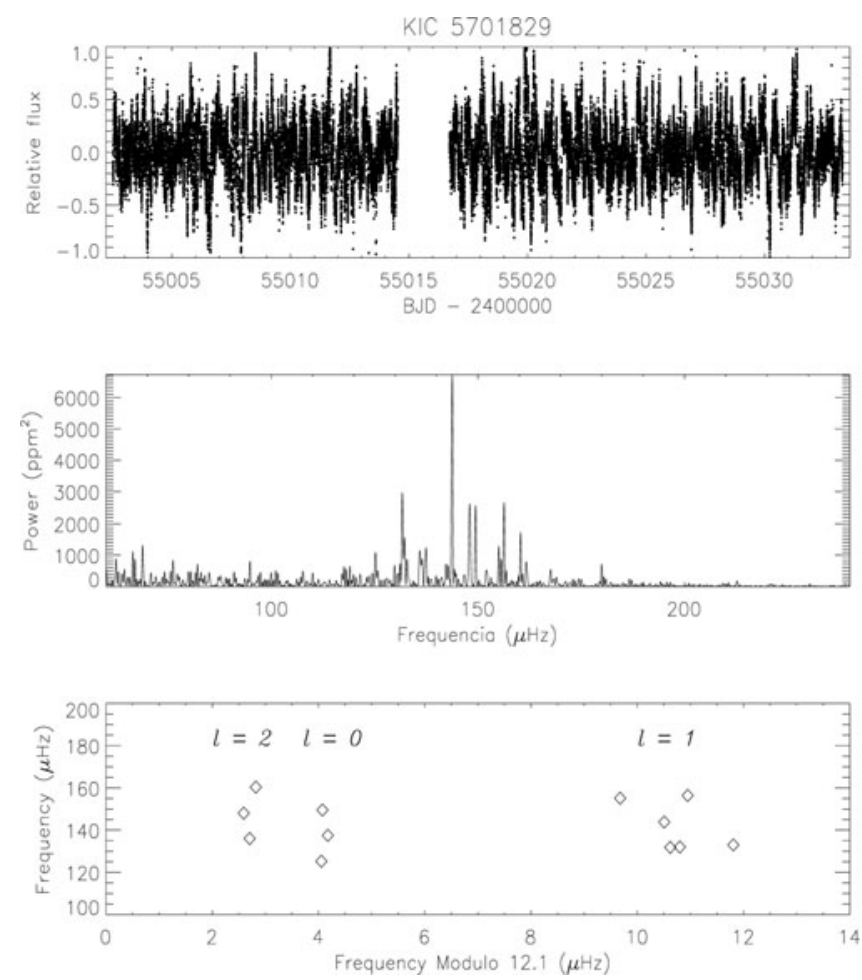

Figure 1. Top panel: KIC 5701829 light curve normalized to the mean value. Middle panel: Fourier spectrum limited to the range of stellar pulsations. Bottom panel: échelle diagram.

\section{Light curve analysis and power spectrum characterization}

KIC 5701829 (= BD+40 3689) was observed with continuous 1-min exposures from BJD 2455002.512255 TO 2455033.303123 for a total of 41823 data points. The raw time series data have been processed as explaining in Baran et al. (2011). The light curve normalized to the mean value are shown in top panel of Fig. 1.The Fourier analysis of the data set has been performed by using the PERIOD04 package Lenz \& Breger (2005). The power spectrum of KIC 5701829 around the highest peak is shown in middle panel of Fig. 1. The resulting power spectrum of KIC 5701829 shows a clear power excess in the frequency range of $100-200 \mu \mathrm{Hz}$ with regular spaced peaks. As well know, the solar-like oscillation spectra are characterized by the large and small frequency separations. The former consists in a series of equally spaced peaks separated by $\Delta \nu$ between $p$ modes of the same degree and adjacent $n: \Delta \nu \sim \Delta \nu_{l}=\nu_{n+1, l}-\nu_{n, l}$. The latter is seen as another series of peaks, with separation of $\delta_{l, l+2}=\nu_{n, l}-\nu_{n-1, l+2}$. While the large frequency separation is proportional to the square root of the mean density, the small frequency separation is sensitive to the chemical composition gradient in the central regions of the star and hence to its evolutionary state. The detected frequencies with an $\mathrm{S} / \mathrm{N}$ ratio in amplitude greater than 3 are shown in Table 1 . The identifications of the modes in terms of the degree $l$ listed in Table 1 are based on the alignment in the échelle diagram which is shown in bottom panel of Fig. 1.

The power spectrum converted to power spectral density is shown in Fig 2 (lightgrey lines). The classical phenomenological description of an oscillation power excess is described by means a Gaussian profile superimposed on the background (Michel et al. 2008, Mosser et al. 2012). In this scenery the power spectrum is composed of three 
Table 1. Detected frequencies and harmonic degree.

\begin{tabular}{cc}
\hline$\nu \mu \mathrm{Hz}$ & $l$ \\
\hline 137.33 & 0 \\
149.36 & 0 \\
125.18 & 0 \\
143.69 & 1 \\
131.66 & 1 \\
156.31 & 1 \\
155.03 & 1 \\
131.89 & 1 \\
132.93 & 1 \\
147.86 & 2 \\
160.24 & 2 \\
135.94 & 2 \\
\hline
\end{tabular}

components: the background, which is assumed to be dominated by stellar noise at low frequency, the oscillation signal centered at $\nu_{\max }$ and the white noise. Following Di Mauro et al. (2011), the complete spectrum was modeled by a superposition of white noise, two semi-Lorentzian functions and a gaussian function representing the power excess hump:

$$
P(\nu)=\sum_{i=1}^{2} \frac{a_{i}}{\left(1+\nu b_{i}\right)^{c_{i}}}+P \exp \left(-\frac{\left(\nu_{\max }-\nu\right)^{2}}{2 \sigma}\right)+W
$$

where $\nu$ is the frequency; $a_{i}, b_{i}$ and $c_{i}$ are the amplitudes, the characteristic time-scales and the slopes of the power laws; $\sigma$ is the width of the power excess hump; $W$ is the contribution of the white noise; $P$ and $\nu_{\max }$ correspond to the height and the location of the maximum oscillation signal, respectively. The IDL least-squares MPFIT package of the Levenberg-Marquardt fitting algorithm has been implemented. The results are shown in Fig 2. The model described by (2.1) was fitted to a smoothed power density spectrum (dark grey line). The resulting central frequency is $\nu_{\max }=148.3 \pm 2.0 \mu \mathrm{Hz}$, where the uncertainty is given by the resolution of the smoothed spectrum. The mean large frequency separation $\Delta \nu$ was calculated by performing a linear fit to the three $l=0$ modes listed in Table 1 . The slope of the fitted line gave the large frequency separation to be $\Delta \nu=12.1 \pm 0.5$.

\section{Discussion}

Molenda-Żakowicz et al. (2013) list atmospheric parameters for KIC 5701829 derived from high resolution spectra with ROTFIT code: $T_{\text {eff }}=4927 \pm 104, \log g=3.19 \pm 0.22$, $[\mathrm{Fe} / \mathrm{H}]=-0.24 \pm 0.21$. KIC 5701829 as expected for red giant stars seems to be a lowrotator with $v \sin i=2 \mathrm{~km} / \mathrm{s}$. Considering the effective temperature the star luminosity is estimated to be $\sim 19 L_{\odot}$. Considering the derived global asteroseismic parameters and the star luminosity it follows that KIC 5701829 is a low-luminosity red giant star. Such stars generally are H-shell-burning stars, have luminosities below about $30 L_{\odot}$ and $\Delta \nu>100 \mu \mathrm{Hz}$ (Bedding et al. 2010). The presence of mixed modes with $l=1$ in KIC 5701829 cannot be discarded due to the scatter seen in the $l=1$ ridge of the échelle diagram.

The empirical scaling relations of Kjeldsen \& Bedding (1995) can be used to provide a first estimate of stellar radius and mass without any stellar modeling: 


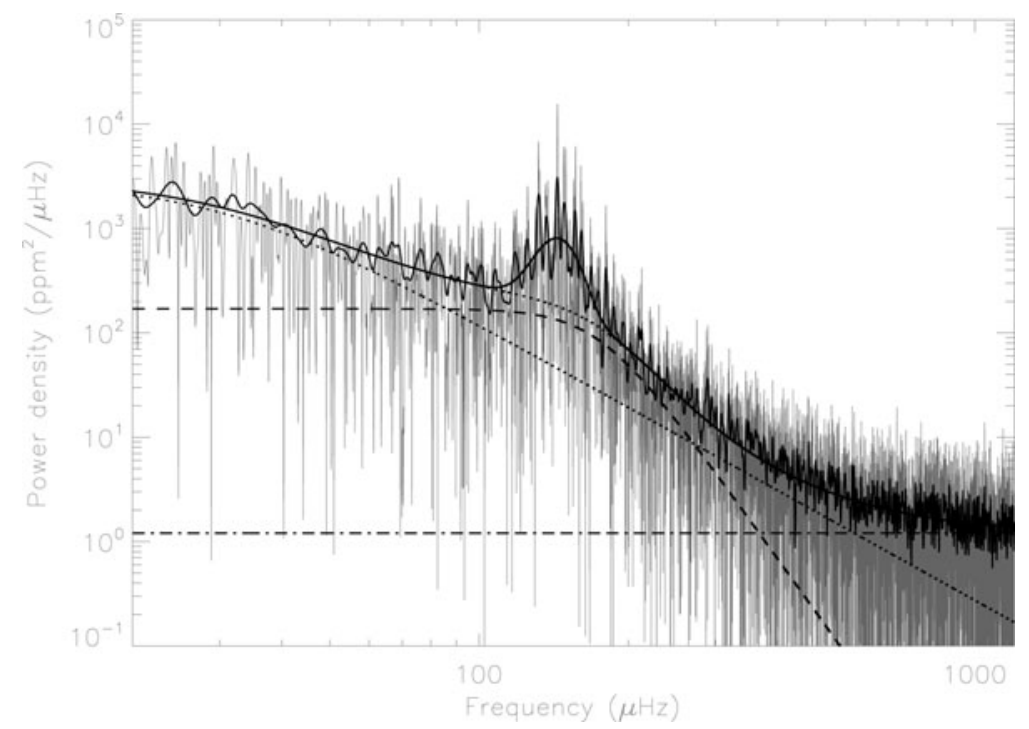

Figure 2. Power spectrum of KIC 5701829. Light grey: power density spectrum; dark grey: the raw spectrum slightly smoothed; thick solid line: global fit according to the model described in the text; dashed, dotted and dash-dotted lines: the two semi-Lorentzians and the white noise component of the global fit, respectively.

$$
\begin{aligned}
\frac{R}{R_{\odot}} & \approx\left(\frac{135 \mu \mathrm{Hz}}{\langle\Delta \nu\rangle}\right)^{2}\left(\frac{\nu_{\max }}{3050 \mu \mathrm{Hz}}\right)\left(\frac{T_{\text {eff }}}{5777 \mathrm{~K}}\right)^{1 / 2}, \\
\frac{M}{M_{\odot}} & \approx\left(\frac{135 \mu \mathrm{Hz}}{\langle\Delta \nu\rangle}\right)^{4}\left(\frac{\nu_{\max }}{3050 \mu \mathrm{Hz}}\right)^{3}\left(\frac{T_{\text {eff }}}{5777 \mathrm{~K}}\right)^{3 / 2} .
\end{aligned}
$$

We have obtained $\frac{M}{M_{\odot}} \simeq 1.52$ and $\frac{R}{R_{\odot}} \simeq 6.05$. This star will be studied in more details including not only the long-cadence observations which with longer timespan yield better frequency resolution, but also stellar modelling to retrieve more precise stellar parameters. This work has received financial support from the UNAM under grants PAPIIT IN 105115 and 106615 .

\section{References}

Aerts, C., Christensen-Dalsgaard, J., \& Kurtz, D. W. 2010, Asteroseismology, Springer Science+Business Media B.V.

Baran, A., Fox Machado, L., Lykke, J., Nielsen, M., \& Telting, J. H. 2011, AcA, 61, 325

Bedding, T. R., Huber, D., Stello, D., et al. 2010, ApJL, 713 , L176

Beck, P. G., Bedding, T. R., Mosser, B., et al. 2011, Science, 332, 205

Borucki, W. J., Koch, D., Basri, G., et al. 2010, Science, 327, 977

Di Mauro, M. P., Cardini, D., Catanzaro, G., et al. 2011, MNRAS, 415, 378

Kjeldsen, H. \& Bedding, T. R. 1995, A\& A, 293, 87

Hekker, S., Gilliland, R. L., Elsworth, Y., et al. 2011, MNRAS, 414, 2594

Lenz, P., \& Breger, M. 2005 , CoAst, 146, 53

Michel, E., Baglin, A., Auvergne, M., et al. 2008, Science, 322, 558

Molenda-Żakowicz, J., Souza, S. G., Frasca, A., et al. 2013, MNRAS, 434, 1422

Mosser, B., Elsworth, Y., Hekker, S., et al. 2012, A\& $A$, 537, A30

Uytterhoeven, K., Moya, A., Grigahcène, A., et al. 2011, A\&AA, 534, A125 